\title{
Modeling and Optimization of Stochastic Joint Replenishment and Delivery Scheduling Problem with Uncertain Costs
}

\author{
Lin Wang, ${ }^{1}$ Hui Qu, ${ }^{1}$ Yanhui $\mathrm{Li}^{2}$ and Jing $\mathrm{He}^{1}$ \\ ${ }^{1}$ School of Management, Huazhong University of Science and Technology, Wuhan 430074, China \\ ${ }^{2}$ School of Information Management, Central China University, Wuhan 430079, China \\ Correspondence should be addressed to Yanhui Li; yhlee@mail.ccnu.edu.cn
}

Received 26 March 2013; Accepted 16 July 2013

Academic Editor: Mustapha Ait Rami

Copyright (c) 2013 Lin Wang et al. This is an open access article distributed under the Creative Commons Attribution License, which permits unrestricted use, distribution, and reproduction in any medium, provided the original work is properly cited.

\begin{abstract}
The stochastic joint replenishment and delivery scheduling (JRD) problem is a key issue in supply chain management and is a major concern for companies. So far, all of the work on stochastic JRDs is under explicit environment. However, the decision makers often have to face vague operational conditions. We develop a practical JRD model with stochastic demand under fuzzy backlogging cost, fuzzy minor ordering cost, and fuzzy inventory holding cost. The problem is to determine procedures for inventory management and vehicle routing simultaneously so that the warehouse may satisfy demand at a minimum long-run average cost. Subsequently, the fuzzy total cost is defuzzified by the graded mean integration representation and centroid approaches to rank fuzzy numbers. To find optimal coordinated decisions, a modified adaptive differential evolution algorithm (MADE) is utilized to find the minimum long-run average total cost. Results of numerical examples indicate that the proposed JRD model can be used to simulate fuzzy environment efficiently, and the MADE outperforms genetic algorithm with a lower total cost and higher convergence rate. The proposed methods can be applied to many industries and can help obtaining optimal decisions under uncertain environment.
\end{abstract}

\section{Introduction}

The joint replenishment problem (JRP) has been heavily researched since the early work of Shu [1]. The JRP means to group items into the same order from a supplier to achieve the purpose of sharing the main preparation costs and saving the procurement costs (Goyal [2]). The existing research of JRPs can be classified into two categories: (1) the classic JRPs under constant demand, (2) JRPs under stochastic or dynamic demand. Corresponding review is available in Khouja and Goyal [3] and Robinson et al. [4].

Supply chain management has received much attention and global sourcing has been widespread in recent years. Since cost savings can be achieved by using joint replenishment policy, managers have realized that jointly considering JRP and delivery scheduling (JRD) can obtain a scale effect of replenishment and transportation simultaneously, thus further reducing total cost (Qu et al. [5], Wang et al. [6]). Unfortunately, the literature of JRD is limited and most of scholars studied JRDs with deterministic demand. Sindhuchao et al. [7] developed the inventory and transportation policy for the central warehouse and decentralized suppliers. They considered the transportation capacity and transportation frequency constraints. Chan et al. [8] discussed delivery scheduling after the JRP has been solved and, respectively, designed four different objectives. Cha et al. [9] developed a joint replenishment and transportation model in a onewarehouse, $n$-retailer system based on improved well-known heuristic named RAND and Genetic Algorithm (GA). They also extended their model with constraints and showed the flexibility of GA. Moon et al. [10] proposed two algorithms to determine the joint replenishment and delivery policies from the perspective of a third party warehouse. Unlike the studies above that consider deterministic demand, Qu et al. [5] discussed a multiitem JRD with modified periodic-review policy under stochastic demand. They considered nonlinear transportation cost and proposed an efficient heuristic algorithm to solve the problem. However, all the existing JRD 
models mentioned above assume explicit vital factors, which is too harsh in practice.

In reality, managers often have to make decisions under imprecise operational conditions (Zeng et al. [11]). Uncertainties have been restrained in a variety of ways and fuzzy set theory has a long history for managing inventories. Chen and Chang [12] proposed a fuzzy economic production quantity model with defective productions that cannot be repaired. Wang et al. [13] studied continuous review inventory models in which a fraction of demand is backordered and the remaining fraction is lost during the stock-out period under fuzzy demands. Dey and Chakraborty [14] discussed a single period inventory model under the mixed fuzzy random environment and provided a methodology to handle the model. On the other hand, defuzzification has been a favorite approach in many inventory studies for its simplicity. Defuzzification can easily transfer fuzziness to be explicit without complex analysis (Lin [15]). So, we will also utilize two defuzzification approaches to handle the fuzziness. However, to the best of our knowledge, only a few papers discussed JRD under fuzzy environment (Wang et al. [16]). However, they assume that demand rates are known and constant. It is too ideal and results in the relatively low practical value of the proposed model.

Moreover, it is very difficult to find optimal solutions for the defuzzified model effectively. The JRP has been proven to be an NP-hard problem (Arkin et al., [17]). While optimal solutions are very hard to be obtained, many acceptable solutions are designed in practice. Current approaches for solving JRPs include an iterative algorithm (Goyal [18]), power-oftwo (PoT) policy (Lee and Yao [19]), RAND algorithm (Kaspi and Rosenblatt [20]), evolutionary algorithm (Olsen [21]), branch-and-bound (Robinson and Gao [22]), and simulated annealing metaheuristic (Robinson et al. [23]). For the JRD model, there are more decision variables which will influence each other. Unfortunately, defuzzification also increases the complexity of the problem further. It is more difficult to solve the fuzzy JRD model in generally acceptable time and CPU memory requirements. Several heuristics may give acceptable solutions when the scale of optimization is small. However, it is not easy to find a proper heuristic with a robust performance.

On the other hand, metaheuristics have grown quickly in order to attain better solutions. Among these algorithms, the evolutionary algorithms (EAs) and especially genetic algorithm (GA) have been proved to be effective algorithms for the JRD. For example, Cha et al. [9] designed two heuristics and a hybrid GA. Results showed the GA outperformed the best solution of each problem in their experiment (the maximum error found is $0.3660 \%$ and the average error found is only $0.0268 \%$ ). However, the GA displays inherent difficulties in performing local search for some numerical applications and the optimal solution cannot be obtained. So, it is necessary to find a novel algorithm to deal with the fuzzy JRD more effectively.

The aim of this paper is to model and optimize the practical JRD policy with stochastic demands under uncertainty. This topic is interesting because of the widely adoption of the JRD policy in many industries and the operability and rationality of the method to handle uncertain costs. This study makes the work of $\mathrm{Qu}$ et al. [5] and Wang et al. [6] become more practical by considering the fuzziness of minor ordering cost, holding cost, and backlogging cost. This study also extends the work of Wang et al. [16] which studied fuzzy JRD under determined environment. The introduction of stochastic demands in the JRD results in rather complex mathematical properties of the proposal model. So, a new approach should be designed to solve this NP-hard problem better. Therefore, we propose a simple and effective modified adaptive differential evolution algorithm (MADE) for the fuzzy JRD model firstly. Results of an example show that an MADE performs better than GA which is also regarded as an effective algorithm for this problem. Then, the MADE is utilized to solve the fuzzy JRD and the results of fuzzy JRD are compared with the JRD. Numerical examples show that the ranges of fuzzy parameters will influence the decision of this problem.

The rest of this paper is organized as follows. In Section 2, we propose a fuzzy JRD model and give the results by two defuzzification approaches. Section 3 proposes an MADE to solve the defuzzified JRD model. Section 4 presents numerical studies and analysis. Section 5 contains the conclusion and provides directions for future research. Preliminaries are presented in the appendix.

\section{Fuzzy JRD Model and Analysis}

\subsection{Stochastic JRD Model with Periodic Review Policy under Crisp}

2.1.1. Problem Description and Notations. We consider the similar situation of a central warehouse (where all stocks are kept) and several geographically dispersed suppliers asserted by Qu et al. [5] and Wang et al. [6]. The central warehouse replenishes its stock by dispatching vehicles to collect the goods from vendors, on routes which begin at the warehouse and end there. When all goods are jointly ordered, one main difficulty faced by the central warehouse is to decide the delivery scheduling in each basic replenishment cycle so that the long-run distribution cost is as low as possible. Blumenfeld et al. [24] described a similar supply network used by General Motors (GMs). Similar issues are also frequently faced by an integrated wholesaler/retailer in the supply of grocery products. The notions used are as follows:
$i$ : the index of items $(i=1,2, \ldots, n)$;
$p$ : the index of suppliers $(p=1,2, \ldots, P)$;
$D_{i}$ : annual demand of item $I$;
$S$ : major ordering cost associated with each order;
$s_{i}$ : minor ordering cost of item $I$;
$h_{i}$ : annual inventory holding cost of item $I$;
$L_{i}$ : the lead time of item $i$;
$R_{i}$ : the maximal inventory level of item $i$ in its replenishment interval;
$z_{i}$ : the tercile of the item $i$ on standard normal distribution (decision variable); 
$\delta_{i}$ : the variance of demand fluctuations per unit time of item $i$;

$W$ : the basic interval after all items replenished jointly, means the lowest common multiple of $\left(k 1, k 2, \ldots, k_{n}\right)$;

$\tau_{i}$ : the shortage cost of item $i$;

$c$ : the unit distance cost of distribution;

$F_{p}$ : the stopover cost in supplier $p$;

$j$ : the index of replenishment period $(j=1,2, \ldots, W)$;

$d(j)$ : the shortest distribution path in the $j$ th replenishment period;

TC: the total annual cost;

$T$ : the basic replenishment cycle per year (decision variable);

$k_{i}$ : the order interval of the item $i$ (decision variable).

Sometimes, the stock-out factor $z_{i}$ is predetermined by decision maker. However, in order to obtain the optimal value of $z_{i}$ or service level, Eynan and Kropp [25] regarded $z_{i}$ as a decision variable, which is the same with the study of $\mathrm{Qu}$ et al. [5]. In this study, we also regard $z_{i}$ as a decision variable.

2.1.2. Formulation of JRD. The total cost consists of the following parts: (1) the inventory cost, which includes ordering, holding, and backlog cost; (2) the transportation cost, which includes dispatching and stopover and routing costs. The details of different costs will be discussed as follows.

(1) Inventory Cost. The ordering cost consists of the major ordering cost which is independent of the number of different items in the order and the minor ordering cost which depends on the number of different items in the order. Hence, the annual ordering cost is

$$
C_{O}=\frac{S}{T}+\sum_{i=1}^{n} \frac{s_{i}}{k_{i} T}
$$

We suppose the demand of each item follows the normal distribution and the lead time is $L_{i}$. As described by Eynan and Kropp [25] and Qu et al. [5], when decision variables $k_{i}$ and $T$ are determined, the demand of item $i$ will be normally distributed in each replenishment interval $\left(k_{i} T+L_{i}\right)$, and the expectation $E$ is equal to $D_{i}\left(k_{i} T+L_{i}\right)$; the variance Var is equal to $\delta_{i}\left(k_{i} T+L_{i}\right)$. Take into the lead time, the maximum inventory $R_{i}$ in each replenishment interval of item $i$ includes the expected demand during the time and the safety stock expressed as a multiple of the standard deviation of demand forecast errors during period $\left(k_{i} T+L_{i}\right)$, that is to say $R_{i}=D_{i}\left(k_{i} T+L_{i}\right)+z_{i} \sqrt{\delta_{i}\left(k_{i} T+L_{i}\right)}$. The average net inventory level of item $i$ during the interval is approximately $R_{i}-D_{i}\left(L_{i}+k_{i} T / 2\right)=(1 / 2) D_{i} k_{i} T+z_{i} \sqrt{\delta_{i}\left(L+k_{i} T\right)}$; thus, the average inventory holding cost for periodic policy is

$$
C_{H}=\sum_{i=1}^{n} h_{i}\left(\frac{1}{2} D_{i} k_{i} T+z_{i} \sqrt{\delta_{i}\left(k_{i} T+L_{i}\right)}\right) \text {. }
$$

A stockout occurs following an order placed at time $t$ if the cumulative demand $x_{i}$ (between $t$ and $t+L_{i}+k_{i} T$ ) exceeds $R_{i}$. Then, there will be a penalty $\operatorname{cost} \tau_{i}$ for each unit backlogged. If demand has a density function $f\left(x_{i}, L_{i}+k_{i} T\right)$ over the interval of length $L_{i}+k_{i} T$, the mean backlogging cost in the long run is

$$
C_{S}=\sum_{i=1}^{n}\left(\frac{\tau_{i}}{k_{i} T}\right)\left(\int_{R_{i}}^{\infty}\left(x_{i}-R_{i}\right) f\left(x_{i}, k_{i} T+L_{i}\right) d x_{i}\right)
$$

(2) Transportation Cost. Define $W$ as the lowest common multiple of $k_{i}$. All items will repeat the same replenishment and distribution policy in every other regeneration cycle $W T$. So we only need to consider the replenishment and distribution policy in a regeneration cycle $W T$.

For each replenishment period $j$, the transportation cost includes the costs of stopover (at those plants visited) plus the travelling costs. Over a regeneration cycle, the average transportation cost is

$$
C_{T}=\frac{\sum_{j=1}^{W}\left(\sum_{p=1}^{P} x_{j p} F_{p}+c d(j)\right)}{W T},
$$

where

$$
x_{j p}= \begin{cases}1, & \text { if the items are kept in the supplier } p \\ & \text { in the } j \text { th replenishment period } \\ 0, & \text { otherwise. }\end{cases}
$$

(3) The Objective. Then, the objective is to minimize the total long-run expected cost (TC);

$$
\begin{aligned}
\operatorname{Min} T C= & C_{O}+C_{H}+C_{S}+C_{T} \\
= & \frac{S}{T}+\sum_{i=1}^{n} \frac{s_{i}}{k_{i} T} \\
& +\sum_{i=1}^{n} h_{i}\left(\frac{1}{2} D_{i} k_{i} T+z_{i} \sqrt{\delta_{i}\left(k_{i} T+L_{i}\right)}\right) \\
& +\sum_{i=1}^{n} \frac{\tau_{i}}{k_{i} T}\left(\int_{R_{i}}^{\infty}\left(x_{i}-R_{i}\right) f\left(x_{i}, k_{i} T+L_{i}\right) d x_{i}\right) \\
& +\frac{\sum_{j=1}^{W}\left(\sum_{p=1}^{P} x_{j p} F_{p}+c d(j)\right)}{W T},
\end{aligned}
$$

where

$$
\begin{gathered}
R_{i}=D_{i}\left(k_{i} T+L_{i}\right)+z_{i} \sqrt{\delta_{i}\left(k_{i} T+L_{i}\right)}, \\
f\left(x_{i}, k_{i} T+L_{i}\right)= \\
\frac{1}{\sqrt{2 \pi \cdot \delta_{i}\left(k_{i} T+L_{i}\right)}} \\
\cdot e^{-\left[x_{i}-D_{i}\left(k_{i} T+L_{i}\right)\right]^{2} / 2 \delta_{i}\left(k_{i} T+L_{i}\right)} .
\end{gathered}
$$




\subsection{Fuzzy JRD Involving Fuzzy Minor Ordering Cost, Holding Cost, and Backlogging Cost}

2.2.1. Formulation. As reported in Vujošević et al. [26], an interesting real problem is when human originated data like holding cost and ordering cost are not precisely known but subjectively estimated or linguistically expressed because of the lack of the accurate history data. Suppose that the minor ordering cost $s_{i}$ and holding cost $h_{i}$ are not precisely known. For example, let the linguistic estimates of $s_{i}$ and $h_{i}$ be as follows.

"The minor ordering cost is about 8 dollars per order, but not less than 6 dollars per order nor more than 15 dollars per order."

"The holding cost is between $15 \%$ and $20 \%$ of the average inventory value, but not less than $12 \%$ nor more than 25\%."

These imprecise definements in linguistics are always described by fuzzy numbers. So, fuzzy variables are also utilized to handle the JRD problem under uncertainty. Two popular kinds of fuzzy numbers characterized by triangular and trapezoidal membership functions (MFs) are presented in the appendix. They were widely used to solve uncertainty problem because of their intuitive appeal and their perceived computational efficacy (Pramanik and Biswas [27]; Giachetti and Young [28]). In addition, trapezoidal fuzzy numbers can be obtained by extending triangular fuzzy numbers (TFNs). That is to say, TFNs can be considered as a particular kind of trapezoidal fuzzy numbers (Vujošević et al. [26]; Giachetti and Young [28]). Thus, we treat the minor ordering cost $s_{i}$, holding cost $h_{i}$, and backlogging $\operatorname{cost} \tau_{i}$ as trapezoidal fuzzy numbers in this study; that is,

$$
\begin{aligned}
\widetilde{s}_{i} & =\left[s_{i 1}, s_{i 2}, s_{i 3}, s_{i 4}\right], \\
\tilde{h}_{i} & =\left[h_{i 1}, h_{i 2}, h_{i 3}, h_{i 4}\right], \\
\tilde{\tau}_{i} & =\left[\tau_{i 1}, \tau_{i 2}, \tau_{i 3}, \tau_{i 4}\right] .
\end{aligned}
$$

According to Definition A.4 in the appendix, the MFs of $\widetilde{s}_{i}, \widetilde{h}_{i}$, and $\widetilde{\tau}_{i}$ are as follows:

$$
\begin{gathered}
\mu_{\widetilde{s}_{i}}(x)= \begin{cases}\frac{x-s_{i 1}}{s_{i 2}-s_{i 1}}, & s_{i 1}<x \leq s_{i 2} \\
1, & s_{i 2}<x \leq s_{i 3} \\
\frac{s_{i 4}-x}{s_{i 4}-s_{i 3}}, & s_{i 3}<x \leq s_{i 4} \\
0, & x \leq s_{i 1} \text { or } x>s_{i 4}\end{cases} \\
\mu_{\tilde{h}_{i}}(x)= \begin{cases}\frac{x-h_{i 1}}{h_{i 2}-h_{i 1}}, & h_{i 1}<x \leq h_{i 2} \\
1, & h_{i 2}<x \leq h_{i 3} \\
\frac{h_{i 4}-x}{h_{i 4}-h_{i 3}}, & h_{i 3}<x \leq h_{i 4} \\
0, & x \leq h_{i 1} \text { or } x>h_{i 4}\end{cases}
\end{gathered}
$$

$$
\mu_{\widetilde{\tau}_{i}}(x)= \begin{cases}\frac{x-\tau_{i 1}}{\tau_{i 2}-\tau_{i 1}}, & \tau_{i 1}<x \leq \tau_{i 2} \\ 1, & \tau_{i 2}<x \leq \tau_{i 3} \\ \frac{\tau_{i 4}-x}{\tau_{i 4}-\tau_{i 3}}, & \tau_{i 3}<x \leq \tau_{i 4} \\ 0, & x \leq \tau_{i 1} \text { or } x>\tau_{i 4} .\end{cases}
$$

Take each TFN into (6) to get the fuzzy number of TC. For the simplify, we denote that

$$
\begin{gathered}
A_{i}=\left(\frac{1}{2} D_{i} k_{i} T+z_{i} \sqrt{\delta_{i}\left(k_{i} T+L_{i}\right)}\right) ; \\
B_{i}=\frac{1}{k_{i} T} \int_{R_{i}}^{\infty}\left(x_{i}-R_{i}\right) f\left(x_{i}, k_{i} T+L_{i}\right) d x_{i} ; \\
C=\frac{S}{T}+\frac{\sum_{j=1}^{W}\left(\sum_{p=1}^{P} x_{j p} F_{p}+c d(j)\right)}{W T} .
\end{gathered}
$$

Hence,

$$
\begin{gathered}
\widetilde{T C}=C+\sum_{i=1}^{n} \frac{\widetilde{s}_{i}}{k_{i} T}+\sum_{i=1}^{n} \widetilde{h}_{i} \cdot A_{i}+\sum_{i=1}^{n} \widetilde{\tau}_{i} \cdot B_{i} \\
=\left[C+\sum_{i=1}^{n} \frac{s_{i 1}}{k_{i} T}+\sum_{i=1}^{n} h_{i 1} \cdot A_{i}+\sum_{i=1}^{n} \tau_{i 1} \cdot B_{i},\right. \\
C+\sum_{i=1}^{n} \frac{s_{i 2}}{k_{i} T}+\sum_{i=1}^{n} h_{i 2} \cdot A_{i}+\sum_{i=1}^{n} \tau_{i 2} \cdot B_{i}, \\
C+\sum_{i=1}^{n} \frac{s_{i 3}}{k_{i} T}+\sum_{i=1}^{n} h_{i 3} \cdot A_{i}+\sum_{i=1}^{n} \tau_{i 3} \cdot B_{i}, \\
\left.C+\sum_{i=1}^{n} \frac{s_{i 4}}{k_{i} T}+\sum_{i=1}^{n} h_{i 4} \cdot A_{i}+\sum_{i=1}^{n} \tau_{i 4} \cdot B_{i}\right] \\
=\left[T C_{1}, T C_{2}, T C_{3}, T C_{4}\right] .
\end{gathered}
$$

2.2.2. Defuzzified TC. Defuzzification has been a favorite approach in many inventory studies for its simplicity. Defuzzification can easily transfer fuzziness to be explicit without complex analysis. In this study, two approaches will be utilized to defuzzify the fuzzy total cost.

(1) Graded Mean Integration Representation (GMIR) Approach. Chen and Hsieh [29] introduced the GMIR method based on the integral value of the graded mean $h$-level of the generalized fuzzy number for defuzzifying a generalized fuzzy number. This method is reasonable because it adopts a grade as the important degree of each point of the support set of a fuzzy number for representing the fuzzy number (G. C. Mahata and P. Mahata [30]). So, we 
also use the GMIR approach to defuzzify the fuzzy number. If $\widetilde{A}=\left[a_{1}, a_{2}, a_{3}, a_{4}\right]$, then the defuzzified function of $\widetilde{A}$ is

$$
\begin{aligned}
P(\widetilde{A}) & =\frac{\int_{0}^{1} h\left[(1 / 2)\left(a_{1}+a_{4}+\left(a_{2}-a_{1}-a_{4}+a_{3}\right) h\right)\right] d h}{\int_{0}^{1} h d h} \\
& =\frac{1}{6}\left(a_{1}+2 a_{2}+2 a_{3}+a_{4}\right),
\end{aligned}
$$

where $h$ is an arbitrary value in the interval of the membership function. According to (12) and (11), we can obtain the defuzzified total cost

$$
P(\widetilde{T C})=\frac{1}{6}\left[T C_{1}+2 T C_{2}+2 T C_{3}+T C_{4}\right] .
$$

So, the simplified objective is

$$
\begin{aligned}
\operatorname{Min} P(\widetilde{T C})=\frac{1}{6}[ & 6 C+\sum_{i=1}^{n} \frac{s_{i 1}+2 s_{i 2}+2 s_{i 3}+s_{i 4}}{k_{i} T} \\
& +\sum_{i=1}^{n}\left(h_{i 1}+2 h_{i 2}+2 h_{i 3}+h_{i 4}\right) \cdot A_{i} \\
& \left.+\sum_{i=1}^{n}\left(\tau_{i 1}+2 \tau_{i 2}+2 \tau_{i 3}+\tau_{i 4}\right) \cdot B_{i}\right] .
\end{aligned}
$$

(2) Centroid. Centroid is a widely used approach for ranking fuzzy numbers by distance method based on calculating the centroid point, where distance means from original point to the centroid point (Wang et al. [31]). If $\widetilde{A}=\left[a_{1}, a_{2}, a_{3}, a_{4}\right]$, then the defuzzified function of $\widetilde{A}$ is

$$
\begin{aligned}
C(\widetilde{A})=\frac{1}{3}[ & a_{1}+a_{2}+a_{3}+a_{4} \\
& \left.-\frac{a_{4} a_{3}-a_{1} a_{2}}{\left(a_{4}+a_{3}\right)-\left(a_{1}+a_{2}\right)}\right] .
\end{aligned}
$$

Hence, the simplified objective is expressed as

$$
\begin{aligned}
\operatorname{Min} C(\widetilde{T C})=\frac{1}{3}[ & T C_{1}+T C_{2}+T C_{3}+T C_{4} \\
& \left.-\frac{T C_{4} T C_{3}-T C_{1} T C_{2}}{\left(T C_{4}+T C_{3}\right)-\left(T C_{1}+T C_{2}\right)}\right] .
\end{aligned}
$$

\section{MADE Algorithm for Fuzzy JRD Model}

3.1. Rationales of Using Differential Evolution. Differential evolution algorithm (DE) was proposed by Storn and Price [32] for complex continuous nonlinear, nondifferentiable, and multimodal optimization problem. This technique combines simple arithmetic operators with the classical events of crossover, mutation, and selection to evolve from a randomly generated starting population to a final solution. Due to its simple structure, easy implementation, quick convergence, and robustness, $\mathrm{DE}$ has been turned out to be one of the best evolution algorithms in a variety of fields (Wang et al. [33]; Wee et al. [34]; Zheng and Yamashiro [35]).

However, the mutation factor $F$ in a typical DE is a constant, which is actually difficult to determine. The main role of $F$ is to perturb the individual in order to avoid being trapped in a local optimum. So, if $F$ is too small, the degree of population diversity cannot reach a certain value and it is easy to be premature. Vice versa, if $F$ is too large, the chance to find optimal solution will decrease, and the effectiveness of the algorithm will be very low in large decision space. More description about the value of $F$ effects on evolution algorithm can be found in Storn and Price [32] and Salman et al. [36]. In this study, we use a modified adaptive DE to improve the quality of solutions of the defuzzified JRD. According to our rule, in the early stage of search, $F$ is large enough to keep the population diversity and avoid premature. Then, $F$ is decreased gradually, and in the latter stage $F$ is small enough to remain the excellent individual and to increase the chance to converge to the optimal solution.

3.2. MADE-Based Procedures for Fuzzy JRD. The model aforementioned in Section 2.2.2 can be decomposed into a JRP and a transportation problem. We use MADE to determine the optimal combination of $k_{i}, z_{i}$, and $T$, and then we can obtain a satisfied solution of the annual total cost. The detailed steps are as follows:

Step 1. Representation and initialization. Let $N_{P}$ represent the population size of a target population, and let $x_{t, G}$ denote the $t$ th target individual at iteration $G$.

Because $k_{i}$ is an integer, we set the lower bound $k_{i}^{\mathrm{LB}}=1$, which are clearly the lower bounds of item $i$. For the upper bound $k_{i}^{\mathrm{UB}}$, we use values of 100 to ensure that the optimal solution will not escape according to the related experience of Cha et al. [9]. According to the properties of the standard normal distribution, we have $P\left(X \leq z_{i}\right)=1-P\left(X>z_{i}\right)=$ 0.995 when $z_{i}=2.575$. So, the range of $z_{i}$ is set as $\left[Z^{\mathrm{LB}}, Z^{\mathrm{UB}}\right]$ $=[0,4]$ which will cover more than $99.99 \%$ of the demand.

According to the related experience of Khouja and Goyal [3] and $\mathrm{Qu}$ et al. [5], $T$ is ranged from 0 to 1 . It is a fact that the annual demand of each item $D_{i}$ is larger than 600 in the following examples; so it is reasonable to assume that at least one item will be replenished per year when $k_{i}$ is not less than 1. That is to say, $T$ should be less than 1 .

By combining $k_{i}, z_{i}$, and $T$, we can obtain the $t$ th individual:

$$
x_{t, G}=\left[k_{i}, z_{i}, T\right]=\left(k_{1}, k_{2}, k_{3}, k_{4}, z_{1}, z_{2}, z_{3}, z_{4}, T\right) .
$$

Then, create the initial population randomly.

Step 2. If the maximum number of iterations is reached, the algorithm will be stopped and output the optimal solution; otherwise, go to the next step. 
Step 3 (mutation). For each target individual $x_{t, G}(i=$ $\left.1,2, \ldots, N_{P}\right)$, a mutant individual $v_{t, G+1}$ is generated according to

$$
v_{t, G+1}=x_{r 1, G}+F \cdot\left(x_{r 2, G}-x_{r 3, G}\right), \quad r 1 \neq r 2 \neq r 3 \neq t .
$$

With randomly chosen integer indexes $r_{1}, r_{2}, r_{3} \in$ $\left\{1,2, \ldots, N_{P}\right\}$. Note that indexes have to be different from each other and from the running index. $F$ is called the mutation factor which controls the amplification of the differential variations $\left(x_{r 2},{ }_{G}, x_{r 3}, G\right)$ and $F$ can be obtained by

$$
F=F_{\min }+\left(F_{\max }-F_{\min }\right) \cdot e^{1-(\mathrm{Gen} M /(\mathrm{Gen} M-G+1))},
$$

where $F_{\min }$ is the minimum of $F$, and $F_{\max }$ is the maximum of $F$. The way to confirm these parameters will be discussed in Section 4.1.

Step 4 (crossover). The crossover operator implements a discrete recombination of the trial individual $v_{t, G+1}$ and the parent individual $x_{t, G}$ to produce offspring $u_{t, G+1}$. The crossover is implemented as follows:

$$
\begin{aligned}
& u_{t l, G+1}= \begin{cases}v_{t l, G+1} & \text { if }(\operatorname{rand}(l) \leq \mathrm{CR}) \text { or } l=\operatorname{rnb}(t) \\
x_{t l, G} & \text { otherwise }\end{cases} \\
& l=1,2, \ldots, D,
\end{aligned}
$$

where $x_{t l}, G$ refers to the $l$ th element of the individual $x_{t, G}$. $u_{t l, G+1}$ and $v_{t l, G+1}$ are similarly defined. rand $(l)$ is the $l$ th evaluation of a uniform random number generator between $[0,1]$. $\mathrm{rnb}(t)$ is a randomly chosen index from $1,2, \ldots, D$ which ensures that $u_{t, G+1}$ gets at least one parameter from $v_{t, G+1}$. Otherwise, no new parent individual would be produced and the population would not alter. CR is the crossover rate between $[0,1]$ which must be determined by the user.

Step 5 (selection). The evaluation function of an offspring is one-to-one competition in the MADE. It means that the resulting trial individual will only replace the original if it has a lower objective function value. Otherwise, the parent will remain in the next generation. The rule is as follows:

$$
x_{t, G+1}= \begin{cases}u_{t, G+1}, & \text { if } g\left(u_{t, G+1}\right) \geq g\left(x_{t, G}\right) \\ x_{t, G}, & \text { if } g\left(u_{t, G+1}\right)<g\left(x_{t, G}\right),\end{cases}
$$

where $g(x)$ means (14) for the GMIR approach or (16) for the centroid approach. This is the same for all variants of the MADE. Then, the best individual of the next generation will be at least as fit as the best individual of the current generation. $G+1$.

Then, the procedure will return to Step 2 after setting $G=$

Step 6. Output the optimal total cost $\mathrm{TC}^{*}$ and the corresponding replenishment cycle $T^{*}$, the order interval $\left(k_{1}, k_{2}, \ldots, k_{n}\right)$, and the tercile $\left(z_{1}, z_{2}, \ldots, z_{n}\right)$.

The steps can be described by the flow chart as shown in Figure 1.

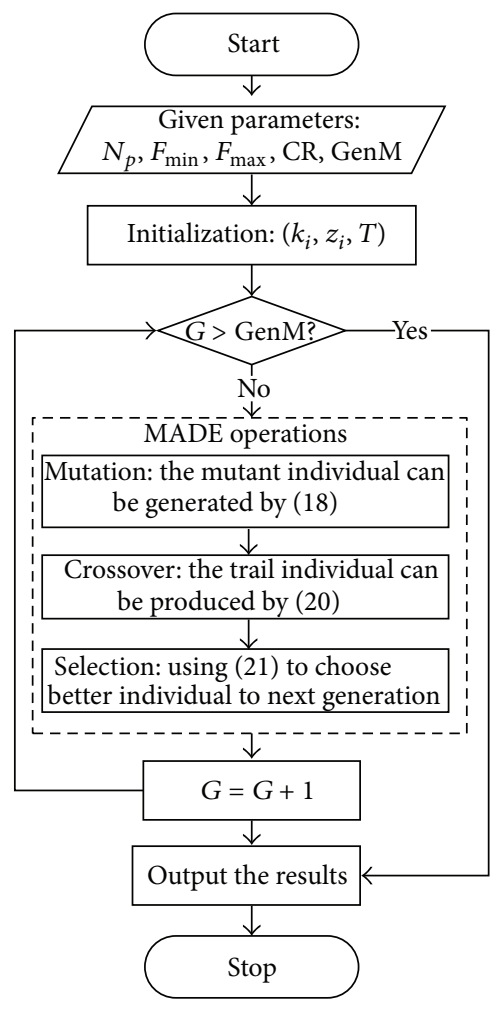

FIGURE 1: Flow chart of the MADE.

3.3. An Example of the Implementation of Operations of $M A D E$. In this section, a simple example of four-product and three-supplier problem is presented in order to better understand the implementation of MADE.

(1) Representation. The sequences of each individual $t$, that is, $x_{t, G}=\left[k_{i}, z_{i}, T\right]$, can be represented as

$$
\begin{array}{lllllllll}
2 & 4 & 5 & 3 & 1.98 & 2.05 & 1.59 & 1.83 & 0.109 .
\end{array}
$$

(2) Mutation (when $F=0.6$ ). For the $t$ th target vector, randomly generate 3 numbers $r_{1}, r_{2}$, and $r_{3}$, which are different from each other and different from $i$.

Sequences of $x_{r 1}, G$

$$
\begin{array}{lllllllll}
3 & 1 & 1 & 2 & 2.09 & 1.49 & 1.08 & 2.39 & 0.098 .
\end{array}
$$

Sequences of $x_{r 2},_{G}$

$$
\begin{array}{lllllllll}
4 & 2 & 3 & 3 & 2.56 & 1.79 & 2.41 & 1.38 & 0.376 .
\end{array}
$$

Sequences of $x_{r 3}, G$

$$
\begin{array}{lllllllll}
2 & 1 & 3 & 2 & 0.68 & 1.09 & 1.89 & 1.76 & 0.289 .
\end{array}
$$

The mutant vector $v_{t, G+1}$ is created by (18):

$$
\begin{array}{lllllllll}
4.2 & 1.6 & 1 & 2.6 & 3.22 & 1.91 & 1.39 & 2.16 & 0.1502 .
\end{array}
$$


TABLE 1: The basic data under deterministic situation.

\begin{tabular}{lcccc}
\hline & Item 1 & Item 2 & Item 3 & Item 4 \\
\hline$s_{i}$ & 25 & 14 & 20 & 30 \\
$D_{i}$ & 600 & 900 & 1200 & 1000 \\
$\delta_{i}$ & 800 & 600 & 700 & 500 \\
$L_{i}$ & 0.02 & 0.02 & 0.02 & 0.02 \\
$h_{i}$ & 5.6 & 21 & 42 & 15 \\
$\tau_{i}$ & 28 & 35 & 40 & 30 \\
\hline
\end{tabular}

TABLE 2: The items supplied by suppliers.

\begin{tabular}{lccc}
\hline & Supplier 1 & Supplier 2 & Supplier 3 \\
\hline Item 1 & 1 & 0 & 0 \\
Item 2 & 0 & 1 & 0 \\
Item 3 & 0 & 0 & 1 \\
Item 4 & 0 & 0 & 1 \\
$F_{p}$ & 40 & 50 & 60 \\
\hline
\end{tabular}

TABLE 3: The distance between the warehouse and suppliers.

\begin{tabular}{lcccc}
\hline & Warehouse & Supplier 1 & Supplier 2 & Supplier 3 \\
\hline Warehouse & 0 & 11 & 9 & 7 \\
Supplier 1 & 11 & 0 & 5 & 8 \\
Supplier 2 & 9 & 5 & 0 & 10 \\
Supplier 3 & 7 & 8 & 10 & 0 \\
\hline
\end{tabular}

(3) Crossover (when $C R=0.3$ ). For each dimension, the randomly generated number rand $(j)$ is

$$
\begin{array}{lllllllll}
0.25 & 0.76 & 0.18 & 0.39 & 0.29 & 0.48 & 0.21 & 0.39 & 0.47
\end{array}
$$

And $\mathrm{rnb}(t)=6$ for this target vector. Therefore, the trial vector $u_{t, G+1}$ is

\section{$\begin{array}{lllllllll}4.2 & 4 & 1 & 3 & 3.22 & 1.91 & 1.39 & 1.83 & 0.109 .\end{array}$}

Since " 4.2 " is not an integer, we round it to the nearest integer; that is, it will be changed to " 4 ." Meanwhile, "3.22" exceeds the range of variety; it is substituted by a number randomly generated from $[0,3]$. After that, the sequences of adjusted $u_{t, G+1}$ are:

\section{$\begin{array}{lllllllll}4 & 4 & 1 & 3 & 1.87 & 1.91 & 1.39 & 1.83 & 0.109 .\end{array}$}

(4) Selection. Since $g\left(u_{t, G+1}\right)=25110, g\left(x_{t, G}\right)=14103$, according to (21), the offspring which will remain to the next generation is:

\section{$\begin{array}{lllllllll}4 & 4 & 1 & 3 & 1.87 & 1.91 & 1.39 & 1.83 & 0.109\end{array}$}

\section{Numerical Examples and Analysis}

In Section 4.1, we will test the performance of MADE with results of GA which was tested to be one of the effective

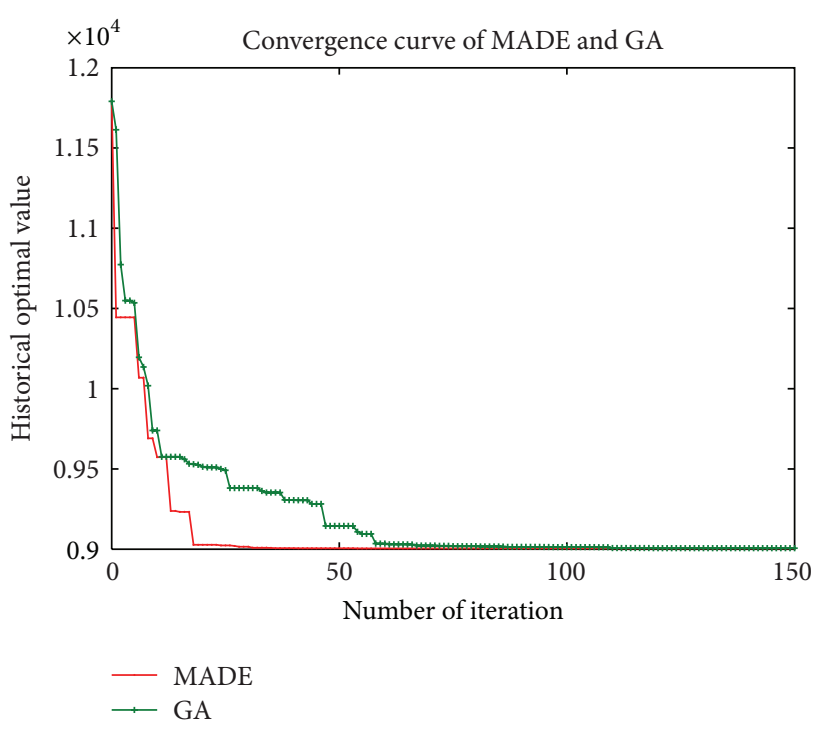

FIgURE 2: Convergence curve of MADE and GA.

algorithms by a stochastic JRD. Then, the MADE will be used to handle the fuzzy JRD.

\subsection{An Example of Stochastic JRD}

(1) Basic Data. The data of the example is the same as $\mathrm{Qu}$ et al. [5]. We suppose that the central warehouse is in charge of the replenishment of 4 items and the inventory management. There are 3 suppliers and the third supplier produces the third and fourth items. The major ordering cost $S$ is 100 , the unit distance cost $c$ is 0.5 , and other data are shown in Tables 1,2 , and 3 , respectively.

(2) Parameters Setting and Results. Storn and Price [32] suggested that $F=0.5, \mathrm{CR}=0.1$ is usually a good initial choice and $N_{P}$ is appropriate for ten times the dimensionality of the problem. Some scholars also provided useful suggestions. According to the recommendation of Neri and Tirronen [37] and Liu and Lampinen [38], we set the relevant parameters of the MADE as follows: $N_{P}=100, F_{\min }=0.2, F_{\max }=1.2$, $\mathrm{CR}=0.1$, and Gen $M=150$. Table 4 shows the result of $\mathrm{Qu}$ et al. [5] in comparison to our result solved by MADE and GA. We programmed the approach of [5], MADE, and GA with the language of Matlab 6.5. Comparison of the CPU time in a computer (memory: 4G; CPU: Intel Core i5-2450 M $2.50 \mathrm{GHz}$ ) by running the example 50 times is also shown in Table 4 .

Both GA and MADE are intelligence EAs. For a perspicuous and direct understanding, we give two convergence curves as shown in Figure 2.

Results listed in Table 4 indicate that the total cost gained by MADE is the smallest and the total cost gotten by GA is the worst. We can also conclude the following: (1) the holding cost of item 1 is 5.6 which is the lowest among all items so that the solved optimal value of $k$ is 2 and the replenishment cycle is the longest; (2) the demand, inventory cost, and shortage cost 
TABLE 4: Results comparison of three approaches.

\begin{tabular}{lcccccc}
\hline & $k_{i}$ & $z_{i}$ & $T$ & TC, min & Convergence rate & CPU time (seconds) \\
\hline Approach of [5] & $3,1,1,1$ & $1.7035,1.7125,1.4060,1.7907$ & 0.0759 & 9021.2 & $/$ & $/$ \\
MADE & $2,1,1,1$ & $1.8537,1.6576,1.3765,1.7441$ & 0.0811 & 9005.9 & $100 \%$ & 4.3734 \\
GA & $2,1,1,1$ & $2.2442,2.1071,1.9193,2.2863$ & 0.0775 & 9213.2 & $66 \%$ & 4.4655 \\
\hline
\end{tabular}

of item 3 are the highest among all items so that we should replenish it in every replenishment cycle.

Obviously, MADE is better than GA and the algorithm of $\mathrm{Qu}$ et al. [5]. So, the MADE will be used to solve the fuzzy JRD.

4.2. Three Scenarios of Fuzzy JRD Model and Analysis. In this section, we will design three different scenarios to compare the results of JRD and fuzzy JRD.

4.2.1. Scenarios 1 . We set the range of the fuzzy set to $\left[d_{1} X\right.$, $\left.d_{2} X, d_{3} X, d_{4} X\right]=[0.7 X, 0.95 X, 1.05 X, 1.1 X]$, where $X$ is the value under certainty. The related data is listed in Table 5.

The major ordering cost $S$ was set to $100,300,500$, and 700 , respectively. The results computed by the MADE under uncertainty and determinacy are shown in Table 6. Here, the average CPU time by running each example 20 times in the abovementioned computer is also reported in Table 6. Statistical test of TC is not provided because MADE is quite effective to find the best TC every time for this problem (maximum error is less than $0.002 \%$ and is regarded as an optimal solution). In the following Scenarios 2 and 3, the CPU time is obtained by the same method and the statistic of TC is also not given for the same reason.

Table 6 shows the following: (1) results of TC under determinacy are larger than TC under uncertainty; (2) $z_{i}$ and $T$ obtained under uncertainty are larger than the corresponding values under determinacy; (3) the best results of TC using GMIR are larger than the corresponding values using centroid; (4) results of $z_{i}$ and $T$ obtained by centroid are larger than GMIR; (5) the CPU times of JRD and fuzzy JRD with GMIR are very close, but the CPU times of fuzzy JRD with centroid are much larger because of the relatively complex computation process.

4.2.2. Scenarios 2 (Symmetrical Fuzzy MFs). Set the range of the fuzzy set $\left[d_{1} X, d_{2} X, d_{3} X, d_{4} X\right]=[0.9 X, 0.95 X, 1.05 X, 1.1$ $X]$. Table 7 shows the data of the ordering cost $\widetilde{s}_{i}$, holding cost $\widetilde{h}_{i}$, and shortage cost $\widetilde{\tau}_{i}$ under uncertainty.

When $S$ was set to $100,300,500$, and 700 , respectively, the results computed by the MADE under uncertainty and determinacy are shown in Table 8.

Results shown in Table 8 indicate that the results of the best TC and $T$ under uncertainty and determinacy are the same for this situation. However, in reality, we are always not able to acquire the accurate value of the parameters or we only can acquire it with very high cost. So, we can use fuzzy theory to solve it reasonably.
4.2.3. Scenarios 3. We extend the range of the fuzzy set to $\left[d_{1} X, d_{2} X, d_{3} X, d_{4} X\right]=[0.9 X, 0.95 X, 1.05 X, 1.3 X]$ and the related data is shown in Table 9.

We set the major ordering cost $S$ to $100,300,500$, and 700 , respectively, and solve the problem in the same way. Table 10 shows the comparison of results under uncertainty and determinacy after extension.

Table 10 indicates that the results of the best TC under uncertainty are larger than the corresponding results of determinacy situation. In comparison with results of $T$ under determinacy, the basic replenishment cycle is smaller.

From Tables 6, 8, and 10, we can reasonably come to the following conclusions:

(1) the ranges of fuzzy parameters will inevitably influence the decision of the JRD. So, it is important to utilize every useful information and to correct judgment to confirm the ranges of fuzzy numbers scientifically and reasonably;

(2) MADE is quite effective for this fuzzy JRD problem because it can find the best minimum long-run average total cost with $100 \%$ convergence rate. The CPU time is also accepted for decision makers.

\section{Conclusion and Future Research}

This paper is an interdisciplinary research of the fuzzy inventory model and intelligent optimization algorithm. A fuzzy JRD model for the one-warehouse, $n$-supplier system is studied while assuming warehouse's minor ordering cost, warehouse's inventory holding cost, and warehouse's storage cost to be fuzzy numbers and a new $\mathrm{DE}$ is proposed. The main contributions are as follows:

(1) Due to the nonavailability of sufficient and precise input data, accurate predicted values of the minor ordering cost, inventory holding cost, and backlogging cost cannot be obtained easily; while fuzzy numbers can efficiently model the imprecise values. Obviously, it is more reasonable to handle imprecise values using the fuzzy theory in the JRD model. It is the first time to introduce fuzziness into the stochastic JRD, which will widen the application field of fuzzy theory and will make the JRD become more practical.

(2) Since the stochastic JRD policy is widely used in many industries, such as manufacturing, wholesale, maintenance, repair, and operating (MRO) supplies, the proposed fuzzy JRD models with two defuzzification methods can also be applied in these industries. 
TABle 5: The basic data after extension.

\begin{tabular}{lccc}
\hline Item & $\widetilde{s}_{i}$ & $\widetilde{h}_{i}$ & $\tilde{\tau}_{i}$ \\
\hline 1 & {$[17.5,23.75,26.25,27.5]$} & {$[3.92,5.32,5.88,6.16]$} & {$[19.6,26.6,29.4,30.8]$} \\
2 & {$[9.8,13.3,14.7,15.4]$} & {$[14.7,19.95,22.05,23.1]$} & {$[24.5,33.25,36.75,38.5]$} \\
3 & {$[14,19,21,22]$} & {$[29.4,39.9,44.1,46.2]$} & {$[28,38,42,44]$} \\
4 & {$[21,28.5,31.5,33]$} & {$[10.5,14.25,15.75,16.5]$} & {$[21,28.5,31.5,33]$} \\
\hline
\end{tabular}

TABLE 6: Results comparison under different situations (scenarios 1).

\begin{tabular}{|c|c|c|c|c|c|c|}
\hline$S$ & Situation & $k_{i}$ & $z_{i}$ & $T$ & $\mathrm{TC}, \min$ & CPU time (seconds) \\
\hline \multirow{3}{*}{100} & Certainty & $(2,1,1,1)$ & $(1.8459,1.6578,1.3710,1.7442)$ & 0.0811 & 9005.90 & 4.3686 \\
\hline & Uncertainty, GMIR & $(2,1,1,1)$ & $(1.8399,1.6514,1.3637,1.7379)$ & 0.0822 & 8805.18 & 4.4798 \\
\hline & Uncertainty, centroid & $(2,1,1,1)$ & $(1.8349,1.6459,1.3575,1.7327)$ & 0.0832 & 8643.61 & 20.1834 \\
\hline \multirow{3}{*}{300} & Certainty & $(2,1,1,1)$ & $(1.7319,1.5343,1.2301,1.6252)$ & 0.1041 & 11165.68 & 4.3143 \\
\hline & Uncertainty, GMIR & $(2,1,1,1)$ & $(1.7249,1.5268,1.2214,1.6179)$ & 0.1057 & 10934.50 & 4.4220 \\
\hline & Uncertainty, centroid & $(2,1,1,1)$ & $(1.7191,1.5205,1.2142,1.6118)$ & 0.1070 & 10747.98 & 20.258 \\
\hline \multirow{3}{*}{500} & Certainty & $(2,1,1,1)$ & $(1.6525,1.4478,1.1302,1.5421)$ & 0.1231 & 12926.66 & 4.2566 \\
\hline & Uncertainty, GMIR & $(2,1,1,1)$ & $(1.6449,1.4396,1.1206,1.5342)$ & 0.1250 & 12668.79 & 4.3772 \\
\hline & Uncertainty, centroid & $(2,1,1,1)$ & $(1.6387,1.4328,1.1127,1.5277)$ & 0.1266 & 12460.48 & 19.8587 \\
\hline \multirow{3}{*}{700} & Certainty & $(1,1,1,1)$ & $(1.8962,1.3601,1.0276,1.4581)$ & 0.1448 & 14415.60 & 4.1407 \\
\hline & Uncertainty, GMIR & $(1,1,1,1)$ & $(1.8893,1.3514,1.0174,1.4497)$ & 0.1471 & 14132.48 & 4.2276 \\
\hline & Uncertainty, centroid & $(1,1,1,1)$ & $(1.8836,1.3442,1.0089,1.4428)$ & 0.1491 & 13903.65 & 19.9156 \\
\hline
\end{tabular}

TABLE 7: The basic data under uncertainty.

\begin{tabular}{lccc}
\hline Item & $\widetilde{s}_{i}$ & $\widetilde{h}_{i}$ & $\tilde{\tau}_{i}$ \\
\hline 1 & {$[22.5,23.75,26.25,27.5]$} & {$[5.04,5.32,5.88,6.16]$} & {$[25.2,26.6,29.4,30.8]$} \\
2 & {$[12.6,13.3,14.7,15.4]$} & {$[18.9,19.95,22.05,23.1]$} & {$[31.5,33.25,36.75,38.5]$} \\
3 & {$[18,19,21,22]$} & {$[37.8,39.9,44.1,46.2]$} & {$[36,38,42,44]$} \\
4 & {$[27,28.5,31.5,33]$} & {$[13.5,14.25,15.75,16.5]$} & {$[27,28.5,31.5,33]$} \\
\hline
\end{tabular}

TABLE 8: Results comparison under different situations (scenarios 2).

\begin{tabular}{ccccccc}
\hline$S$ & Situation & $k_{i}$ & $z_{i}$ & $T$ & TC, min & CPU time (seconds) \\
\hline \multirow{3}{*}{100} & Certainty & $(2,1,1,1)$ & $(1.8459,1.6578,1.3710,1.7442)$ & 0.0811 & 9005.90 & 4.4341 \\
& Uncertainty, GMIR & $(2,1,1,1)$ & $(1.8459,1.6578,1.3710,1.7442)$ & 0.0811 & 9005.90 & 4.6048 \\
& Uncertainty, centroid & $(2,1,1,1)$ & $(1.8459,1.6578,1.3710,1.7442)$ & 0.0811 & 9005.90 & 21.0726 \\
\hline \multirow{2}{*}{300} & Certainty & $(2,1,1,1)$ & $(1.7319,1.5343,1.2301,1.6252)$ & 0.1041 & 11165.68 & 4.3173 \\
& Uncertainty, GMIR & $(2,1,1,1)$ & $(1.7319,1.5343,1.2301,1.6252)$ & 0.1041 & 11165.68 & 4.4136 \\
& Uncertainty, centroid & $(2,1,1,1)$ & $(1.7319,1.5343,1.2301,1.6252)$ & 0.1041 & 11165.68 & 20.3023 \\
\hline \multirow{2}{*}{500} & Certainty & $(2,1,1,1)$ & $(1.6525,1.4478,1.1302,1.5421)$ & 0.1231 & 12926.66 & 4.2955 \\
& Uncertainty, GMIR & $(2,1,1,1)$ & $(1.6525,1.4478,1.1302,1.5421)$ & 0.1231 & 12926.66 & 4.37988 \\
& Uncertainty, centroid & $(2,1,1,1)$ & $(1.6525,1.4478,1.1302,1.5421)$ & 0.1231 & 12926.66 & 19.8634 \\
\hline \multirow{2}{*}{700} & Certainty & $(2,1,1,1)$ & $(1.8962,1.3601,1.0276,1.4581)$ & 0.1448 & 14415.60 & \\
& Uncertainty, GMIR & $(2,1,1,1)$ & $(1.8962,1.3601,1.0276,1.4581)$ & 0.1448 & 14415.60 & 4.1261 \\
& Uncertainty, centroid & $(2,1,1,1)$ & $(1.8962,1.3601,1.0276,1.4581)$ & 0.1448 & 14415.60 & 4.2493 \\
\hline
\end{tabular}

TABLE 9: The basic data after extension.

\begin{tabular}{lccc}
\hline item & $\widetilde{s}_{i}$ & $\widetilde{h}_{i}$ & $\widetilde{\tau}_{i}$ \\
\hline 1 & {$[22.5,23.75,26.25,32.5]$} & {$[5.04,5.32,5.88,7.28]$} & {$[25.2,26.6,29.4,36.4]$} \\
2 & {$[12.6,13.3,14.7,18.2]$} & {$[18.9,19.95,22.05,27.3]$} & {$[31.5,33.25,36.75,45.5]$} \\
3 & {$[18,19,21,26]$} & {$[37.8,39.9,44.1,54.6]$} & {$[36,38,42,52]$} \\
4 & {$[27,28.5,31.5,39]$} & {$[13.5,14.25,15.75,19.5]$} & {$[27,28.5,31.5,39]$} \\
\hline
\end{tabular}


TABLE 10: Results comparison under different situations after extension (scenarios 3).

\begin{tabular}{|c|c|c|c|c|c|c|}
\hline$S$ & Situation & $k_{i}$ & $z_{i}$ & $T$ & $\mathrm{TC}, \mathrm{min}$ & CPU time (seconds) \\
\hline \multirow{3}{*}{100} & Certainty & $(2,1,1,1)$ & $(1.8459,1.6578,1.3710,1.7442)$ & 0.0811 & 9005.90 & 4.3782 \\
\hline & Uncertainty, GMIR & $(2,1,1,1)$ & $(1.8516,1.664,1.378,1.7501)$ & 0.0801 & 9205.31 & 4.5183 \\
\hline & Uncertainty, centroid & $(2,1,1,1)$ & $(1.856,1.6687,1.3833,1.7547)$ & 0.0793 & 9363.95 & 20.6277 \\
\hline \multirow{3}{*}{300} & Certainty & $(2,1,1,1)$ & $(1.7319,1.5343,1.2301,1.6252)$ & 0.1041 & 11165.68 & 4.2908 \\
\hline & Uncertainty, GMIR & $(2,1,1,1)$ & $(1.7386,1.5416,1.2385,1.6322)$ & 0.1026 & 11394.79 & 4.4135 \\
\hline & Uncertainty, centroid & $(2,1,1,1)$ & $(1.7437,1.5472,1.2449,1.6376)$ & 0.1015 & 11576.66 & 20.0418 \\
\hline \multirow{3}{*}{500} & Certainty & $(2,1,1,1)$ & $(1.6525,1.4478,1.1302,1.5421)$ & 0.1231 & 12926.66 & 4.2934 \\
\hline & Uncertainty, GMIR & $(2,1,1,1)$ & $(1.6597,1.4558,1.1393,1.5497)$ & 0.1212 & 13181.85 & 4.3672 \\
\hline & Uncertainty, centroid & $(2,1,1,1)$ & $(1.665,1.4616,1.1461,1.5553)$ & 0.1199 & 13384.18 & 20.1400 \\
\hline \multirow{3}{*}{700} & Certainty & $(1,1,1,1)$ & $(1.8962,1.3601,1.0276,1.4581)$ & 0.1448 & 14415.60 & 4.1279 \\
\hline & Uncertainty, GMIR & $(1,1,1,1)$ & $(1.9029,1.3685,1.0374,1.466)$ & 0.1426 & 14695.63 & 4.2845 \\
\hline & Uncertainty, centroid & $(1,1,1,1)$ & $(1.908,1.3749,1.045,1.4722)$ & 0.1410 & 14917.54 & 19.8705 \\
\hline
\end{tabular}

(3) The proposed simple and effective MADE-based approach can find the optimal cycle time and safety factor of each item for the defuzzified JRD effectively. Moreover, the convergence rate of MADE outperforms another popular GA-based approach. Therefore, managers can always know the corresponding minimum total cost under fuzzy environment, just like the case of precise data. This study expands the application field of the DE.

In the future, a dependent-chance programming model or a chance-constrained programming model can be designed for the JRD model of the one-warehouse, n-retailer system in fuzzy environment. The DE still can provide good solutions to these problems.

\section{Appendix}

In order to solve the fuzzy JRD model, we need to use the following definitions and properties.

Definition A.1. A fuzzy set $\tilde{a}$ on $R=(-\infty,+\infty)$ is called a fuzzy point if its MF is

$$
\mu_{\widetilde{a}}(x)= \begin{cases}1, & x=a \\ 0, & x \neq a,\end{cases}
$$

where point $a$ is called its support.

Let the family of all fuzzy points be $F_{p}=\{\widetilde{a} \mid \forall a \in R\}$. The real number $a \in R$ and the fuzzy point $\tilde{a} \in F_{p}$ are in one-to-one and onto mapping.

Definition A.2. A fuzzy set $\left[a_{x}, b_{x}\right]$ on $R, 0 \leq a \leq 1, a<b$, is called a level $\alpha$ fuzzy interval if its MF is

$$
\mu_{\left[a_{x}, b_{x}\right]}(x)= \begin{cases}\alpha, & a \leq x \leq b \\ 0, & \text { otherwise }\end{cases}
$$

Definition A.3. A fuzzy set $\widetilde{A}=[a, b, c]$ on $R, a<b<c$, is called a triangular fuzzy number if its MF is

$$
\mu_{\widetilde{A}}(x)= \begin{cases}\frac{x-a}{b-a}, & a<x \leq b \\ \frac{c-x}{c-b}, & b<x \leq c \\ 0, & x \leq a, \text { or } x>c .\end{cases}
$$

When $a=b=c$, we have fuzzy point $(c, c, c)=\widetilde{c}$.

Definition A.4. A fuzzy set $\widetilde{A}=[a, b, c, d]$ on $R, a<b<c<$ $d$, is called a trapezoidal fuzzy number if its MF is

$$
\mu_{\widetilde{A}}(x)= \begin{cases}\frac{x-a}{b-a}, & a<x \leq b \\ 1, & b<x \leq c \\ \frac{d-x}{d-c}, & c<x \leq d \\ 0, & x \leq a \text { or } x>d .\end{cases}
$$

\section{Acknowledgments}

This research is partially supported by National Natural Science Foundation of China (70801030, 71371080, 71131004, 70871050, and 71171093), Humanities and Social Sciences Foundation of Chinese Ministry of Education (no. 11YJC630275), and Fundamental Research Funds for the Central Universities (HUST: 2012TS065, and CXY12Q043). The authors are very grateful for their contribution of data handling of Chen Xiao-xi and Dun Cai-xia.

\section{References}

[1] F. T. Shu, "Economic ordering frequency for two items jointly replenished," Management Science, vol. 17, no. 6, pp. B406-B410, 1971.

[2] S. K. Goyal, "Determination of economic packaging frequency for items jointly replenished," Management Science, vol. 20, no. 2, pp. 232-235, 1973. 
[3] M. Khouja and S. Goyal, "A review of the joint replenishment problem literature: 1989-2005," European Journal of Operational Research, vol. 186, no. 1, pp. 1-16, 2008.

[4] P. Robinson, A. Narayanan, and F. Sahin, "Coordinated deterministic dynamic demand lot-sizing problem: a review of models and algorithms," Omega, vol. 37, no. 1, pp. 3-15, 2009.

[5] W. W. Qu, J. H. Bookbinder, and P. Iyogun, "Integrated inventory-transportation system with modified periodic policy for multiple products," European Journal of Operational Research, vol. 115, no. 2, pp. 254-269, 1999.

[6] L. Wang, C. X. Dun, W. J. Bi, and Y. R. Zeng, "An effective and efficient differential evolution algorithm for the integrated stochastic joint replenishment and delivery model," KnowledgeBased Systems, vol. 36, pp. 104-114, 2012.

[7] S. Sindhuchao, H. E. Romeijn, E. Akçali, and R. Boondiskulchok, "An integrated inventory-routing system for multi-item joint replenishment with limited vehicle capacity," Journal of Global Optimization, vol. 32, no. 1, pp. 93-118, 2005.

[8] C. K. Chan, L. Y.-O. Li, C. T. Ng, B. K.-S. Cheung, and A. Langevin, "Scheduling of multi-buyer joint replenishments," International Journal of Production Economics, vol. 102, no. 1, pp. 132-142, 2006.

[9] B. C. Cha, I. K. Moon, and J. H. Park, “The joint replenishment and delivery scheduling of the one-warehouse, n-retailer system," Transportation Research Part E, vol. 44, no. 5, pp. 720-730, 2008.

[10] I. K. Moon, B. C. Cha, and C. U. Lee, "The joint replenishment and freight consolidation of a warehouse in a supply chain," International Journal of Production Economics, vol. 133, no. 1, pp. 344-350, 2011.

[11] Y. R. Zeng, L. Wang, and J. He, "A novel approach for evaluating control criticality of spare parts using fuzzy comprehensive evaluation and GRA," International Journal of Fuzzy Systems, vol. 14, no. 3, pp. 392-401, 2012.

[12] S. H. Chen and S. M. Chang, "Optimization of fuzzy production inventory model with unrepairable defective products," International Journal of Production Economics, vol. 113, no. 2, pp. 887894, 2008.

[13] L. Wang, Q.-L. Fu, and Y.-R. Zeng, "Continuous review inventory models with a mixture of backorders and lost sales under fuzzy demand and different decision situations," Expert Systems with Applications, vol. 39, no. 4, pp. 4181-4189, 2012.

[14] O. Dey and D. Chakraborty, "A single period inventory model with a truncated normally distributed fuzzy random variable demand," International Journal of Systems Science, vol. 43, no. 3, pp. 518-525, 2012.

[15] Y.-J. Lin, "A periodic review inventory model involving fuzzy expected demand short and fuzzy backorder rate," Computers and Industrial Engineering, vol. 54, no. 3, pp. 666-676, 2008.

[16] L. Wang, C. X. Dun, C. G. Lee, Q. L. Fu, and Y. R. Zeng, "Model and algorithm for fuzzy joint replenishment and delivery scheduling without explicit membership function," International Journal of Advanced Manufacturing Technology, vol. 66, no. 9-12, pp. 1907-1920, 2013.

[17] E. Arkin, D. Joneja, and R. Roundy, "Computational complexity of uncapacitated multi-echelon production planning problems," Operations Research Letters, vol. 8, no. 2, pp. 61-66, 1989.

[18] S. K. Goyal, "Determination of optimum packaging frequency of items jointly replenished," Management Science, vol. 21, no. 4, pp. 436-443, 1974.
[19] F.-C. Lee and M.-J. Yao, "A global optimum search algorithm for the joint replenishment problem under power-of-two policy," Computers and Operations Research, vol. 30, no. 9, pp. 1319-1333, 2003.

[20] M. Kaspi and M. J. Rosenblatt, "On the economic ordering quantity for jointly replenished items," International Journal of Production Research, vol. 29, no. 1, pp. 107-114, 1991.

[21] A. L. Olsen, "An evolutionary algorithm to solve the joint replenishment problem using direct grouping," Computers and Industrial Engineering, vol. 48, no. 2, pp. 223-235, 2005.

[22] E. P. Robinson and L.-L. Gao, "A dual ascent procedure for multiproduct dynamic demand coordinated replenishment with backlogging," Management Science, vol. 42, no. 11, pp. 1556-1564, 1996.

[23] E. P. Robinson, A. Narayanan, and L.-L. Gao, "Effective heuristics for the dynamic demand joint replenishment problem," Journal of the Operational Research Society, vol. 58, no. 6, pp. 808-815, 2007.

[24] D. E. Blumenfeld, L. D. Burns, C. F. Daganzo, M. C. Frick, and R. W. Hall, "Reducing logistics costs at general motors," Interfaces, vol. 17, no. 1, pp. 26-47, 1987.

[25] A. Eynan and D. H. Kropp, "Periodic review and joint replenishment in stochastic demand environments," IIE Transactions, vol. 30, no. 11, pp. 1025-1033, 1998.

[26] M. Vujošević, D. Petrović, and R. Petrović, "EOQ gormula when inventory is fuzzy," International Journal of Production Economics, vol. 45, no. 1-3, pp. 499-504, 1996.

[27] S. Pramanik and P. Biswas, "Multi-objective assignment problem with generalized trapezoidal fuzzy numbers," International Journal of Applied Information Systems, vol. 2, no. 6, pp. 13-20, 2012.

[28] R. E. Giachetti and R. E. Young, "Analysis of the error in the standard approximation used for multiplication of triangular and trapezoidal fuzzy numbers and the development of a new approximation," Fuzzy Sets and Systems, vol. 91, no. 1, pp. 1-13, 1997.

[29] S. H. Chen and C. H. Hsieh, "Graded mean integration representation of generalized fuzzy number," Journal of Chinese Fuzzy Systems, vol. 5, no. 2, pp. 1-7, 1997.

[30] G. C. Mahata and P. Mahata, "Analysis of a fuzzy economic order quantity model for deteriorating items under retailer partial trade credit financing in a supply chain," Mathematical and Computer Modelling, vol. 53, no. 9-10, pp. 1621-1636, 2011.

[31] Y.-M. Wang, J.-B. Yang, D.-L. Xu, and K.-S. Chin, "On the centroids of fuzzy numbers," Fuzzy Sets and Systems, vol. 157, no. 7, pp. 919-926, 2006.

[32] R. Storn and K. Price, "Differential evolution-a simple and efficient heuristic for global optimization over continuous spaces," Journal of Global Optimization, vol. 11, no. 4, pp. 341359, 1997.

[33] L. Wang, J. He, and Y.-R. Zeng, "A differential evolution algorithm for joint replenishment problem using direct grouping and its application," Expert Systems, vol. 29, no. 5, pp. 429-441, 2012.

[34] H.-M. Wee, C.-C. Lo, and P.-H. Hsu, "A multi-objective joint replenishment inventory model of deteriorated items in a fuzzy environment," European Journal of Operational Research, vol. 197, no. 2, pp. 620-631, 2009.

[35] T. Zheng and M. Yamashiro, "Solving flow shop scheduling problems by quantum differential evolutionary algorithm," International Journal of Advanced Manufacturing Technology, vol. 49, no. 5-8, pp. 643-662, 2010. 
[36] A. Salman, A. P. Engelbrecht, and M. G. H. Omran, "Empirical analysis of self-adaptive differential evolution," European Journal of Operational Research, vol. 183, no. 2, pp. 785-804, 2007.

[37] F. Neri and V. Tirronen, "Recent advances in differential evolution: a survey and experimental analysis," Artificial Intelligence Review, vol. 33, no. 1-2, pp. 61-106, 2010.

[38] J. Liu and J. Lampinen, "On setting the control parameter of the differential evolution algorithm," in Proceedings of the 8th International Mendel Conference on Soft Computing, pp. 11-18, 2002. 


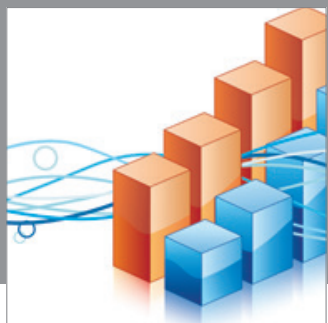

Advances in

Operations Research

mansans

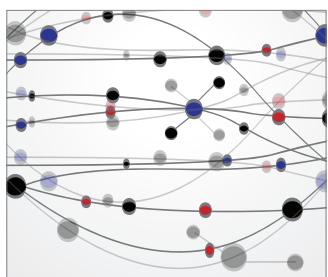

The Scientific World Journal
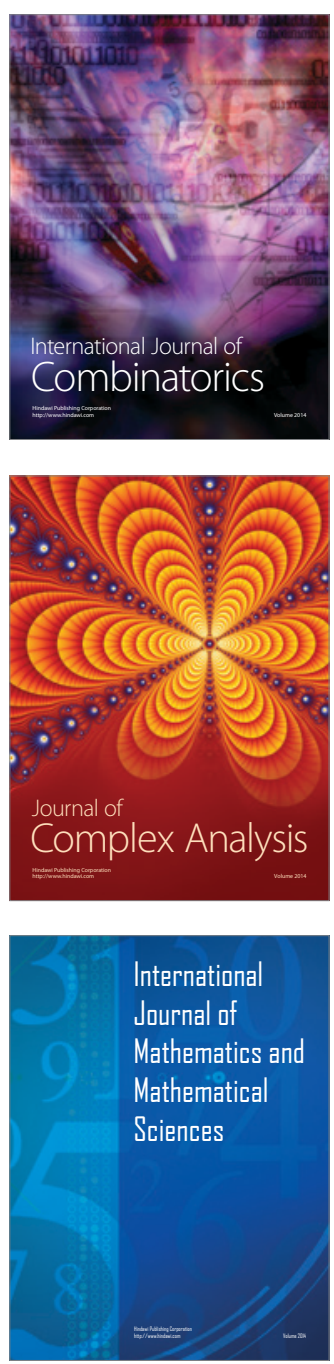
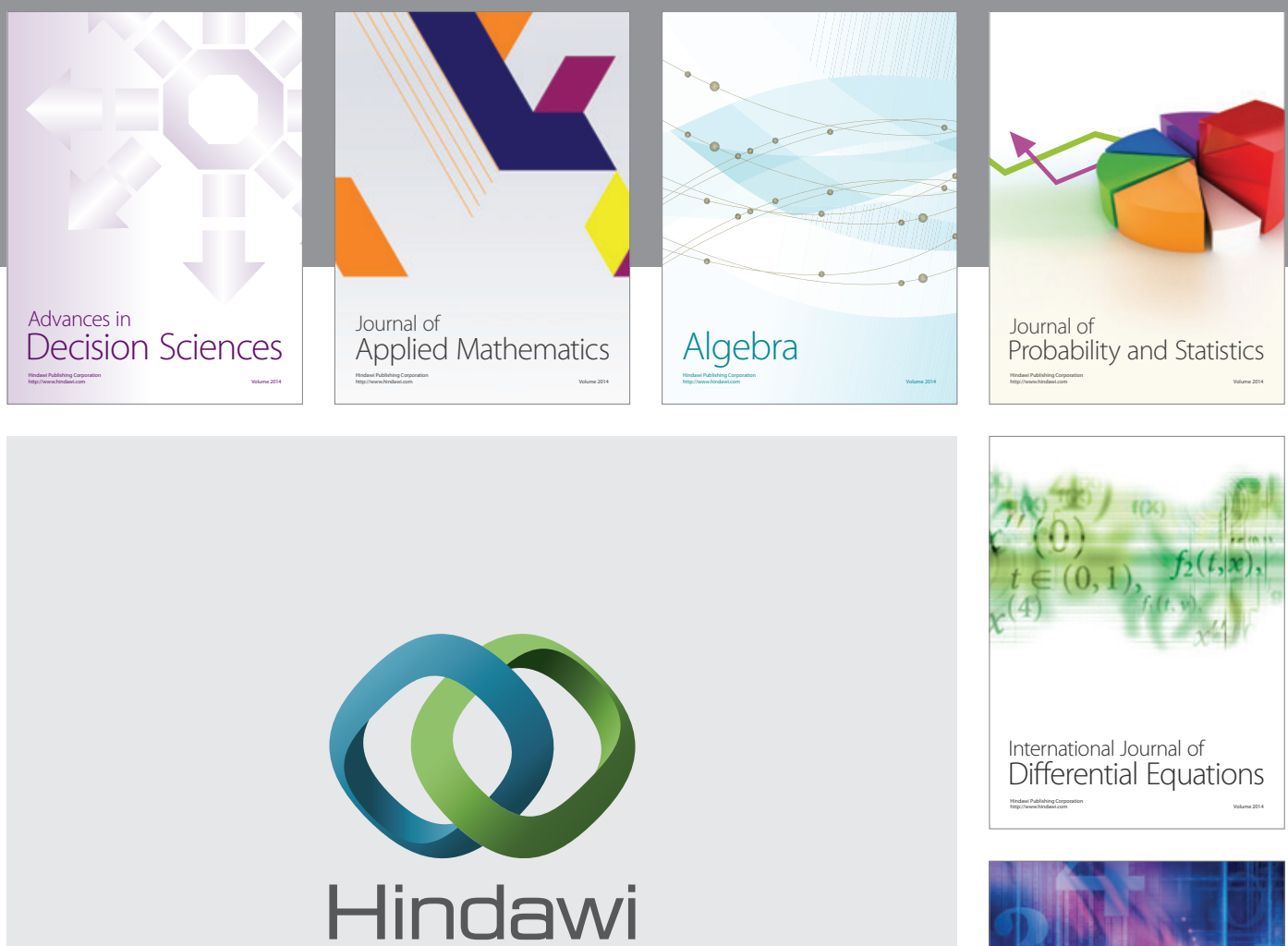

Submit your manuscripts at http://www.hindawi.com
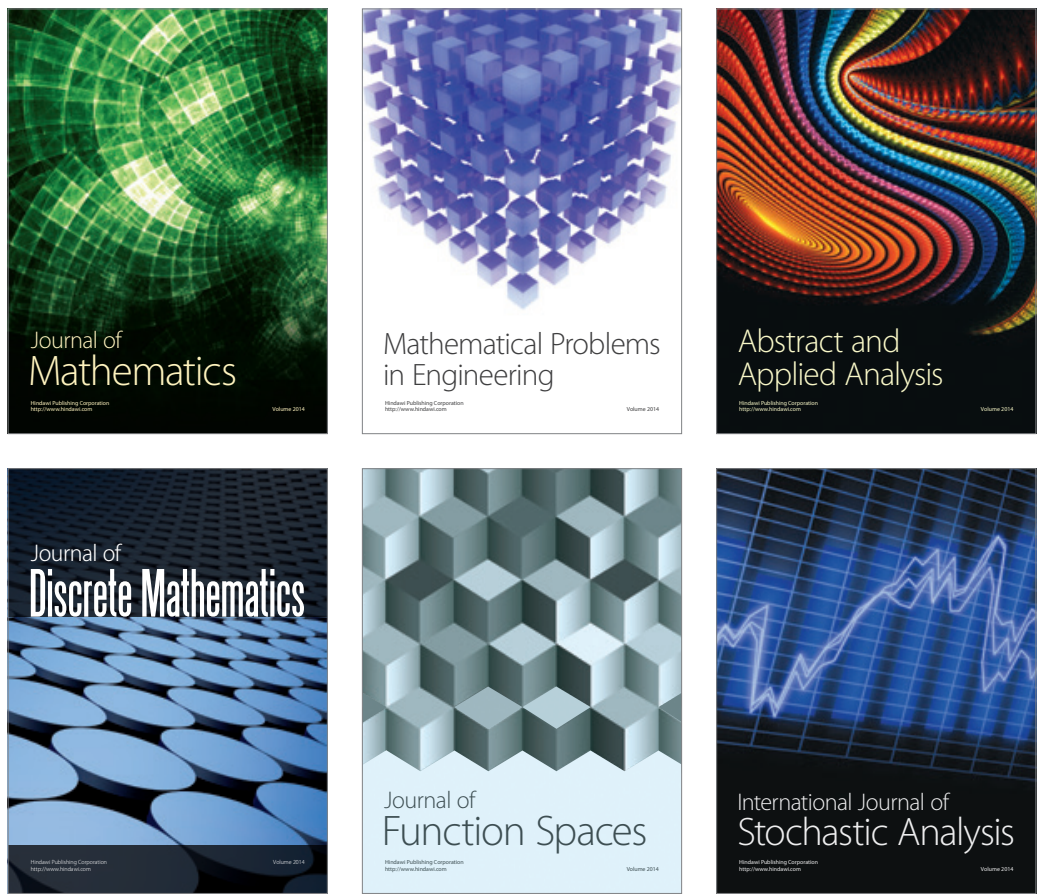

Journal of

Function Spaces

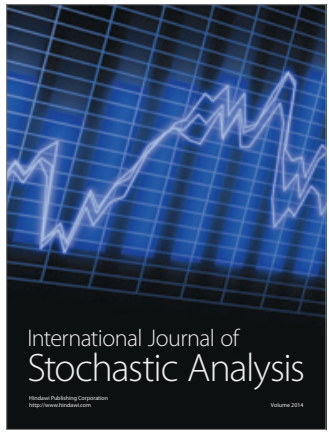

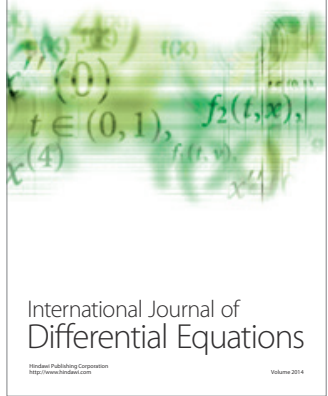
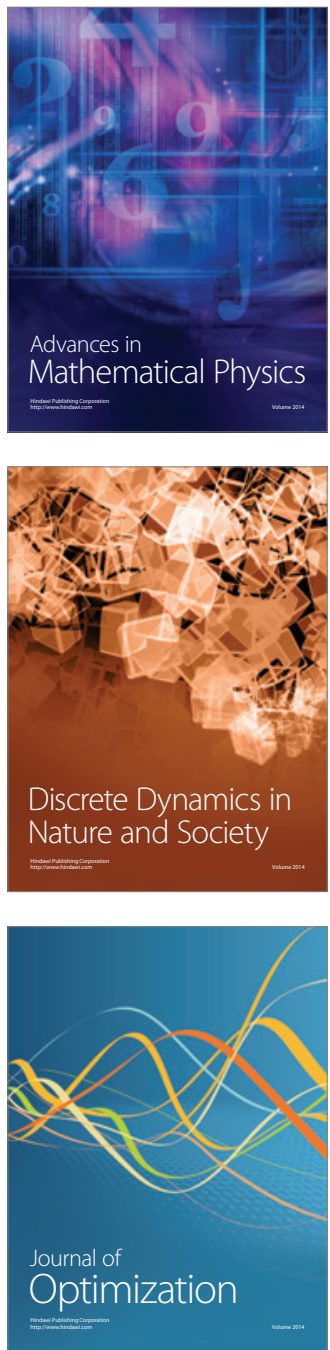